\title{
On Chen invariants and inequalities in quaternionic geometry
}

\section{Gabriel-Eduard Vîlcu*}

\section{"Correspondence:}

gvilcu@upg-ploiesti.ro;

gvilcu@gta.math.unibuc.ro

Department of Mathematical

Modelling, Economic Analysis and

Statistics, Petroleum-Gas University

of Ploieşti, Bulevardul Bucureşti

Nr. 39, Ploiești, 100680, Romania

Faculty of Mathematics and

Computer Science, Research Center

in Geometry, Topology and Algebra,

University of Bucharest, Str.

Academiei, Nr. 14, Sector 1,

Bucureşti, 70109, Romania

\begin{abstract}
In this paper we give a survey of main results concerning Chen inequalities for submanifolds in quaternionic space forms and propose some open problems in the field for further research.
\end{abstract}

AMS Subject Classification: 53C15; 53C25; 53C40

Keywords: Chen's invariant; squared mean curvature; shape operator; quaternionic space form; slant submanifold

\section{Introduction}

The theory of Chen invariants, initiated by Prof. B.-Y. Chen in a seminal paper published in 1993 [1], is presently one of the most interesting research topic in differential geometry of submanifolds. The author's original motivation to introduce new types of Riemannian invariants, known as $\delta$-invariants or Chen invariants, was the need to provide answers to an open question raised by Chern concerning the existence of minimal immersions into a Euclidean space of arbitrary dimension [2]. In fact, due the lack of control of the extrinsic properties of the submanifolds by the known intrinsic invariants, no solutions to Chern's problem were known before the invention of Chen invariants (see [3]). Therefore, in [1], Chen obtained a necessary condition for the existence of minimal isometric immersion from a given Riemannian manifold $M$ into Euclidean space and established a sharp inequality for a submanifold in a real space form using the scalar curvature and the sectional curvature (both being intrinsic invariants) and squared mean curvature (the main extrinsic invariant). On the other hand, in [4], Chen obtained inequalities between the $k$-Ricci curvature, the squared mean curvature and the shape operator for submanifolds in real space forms with arbitrary codimensions. These inequalities are also sharp, and many nice classes of submanifolds realize equality in all above inequalities. Since then many papers concerning Chen invariants and inequalities have appeared in the literature for different classes of submanifolds in various ambient spaces, like complex space forms [5-7], cosymplectic space forms [8-10], Sasakian space forms [11-13], locally conformal Kähler space forms [14-16], generalized complex space forms [17-19], locally conformal almost cosymplectic manifolds [20, 21], $(\kappa, \mu)$-contact space forms [22, 23], Kenmotsu space forms [24, 25], $S$-space forms [26, 27], $T$-space forms [28]; see also [29] and references therein.

In the last decade, Chen-like inequalities were extended in the quaternionic setting. It is the main purpose of this paper to present the evolution of these inequalities for sub-

(c) 2013 Vîlcu; licensee Springer. This is an Open Access article distributed under the terms of the Creative Commons Attribution License (http://creativecommons.org/licenses/by/2.0), which permits unrestricted use, distribution, and reproduction in any medium, provided the original work is properly cited. 
manifolds in quaternionic space forms, to survey some recent results on this topic and to propose a set of natural problems in the field.

\section{Preliminaries}

\subsection{Riemannian invariants}

In this subsection we recall some basic concepts concerning Chen invariants, using mainly [30].

Let $M$ be an $n$-dimensional Riemannian manifold. We denote by $K(\pi)$ the sectional curvature of $M$ associated with a plane section $\pi \subset T_{p} M, p \in M$. If $\left\{e_{1}, \ldots, e_{n}\right\}$ is an orthonormal basis of the tangent space $T_{p} M$, the scalar curvature $\tau$ at $p$ is defined by

$$
\tau(p)=\sum_{1 \leq i<j \leq n} K\left(e_{i} \wedge e_{j}\right)
$$

One denotes

$$
(\inf K)(p)=\inf \left\{K(\pi) \mid \pi \subset T_{p} M, \operatorname{dim} \pi=2\right\}
$$

and the Chen first invariant is given by

$$
\delta_{M}(p)=\tau(p)-(\inf K)(p) .
$$

Suppose $L$ is an $r$-dimensional subspace of $T_{p} M, r \geq 2$ and $\left\{e_{1}, \ldots, e_{r}\right\}$ an orthonormal basis of $L$. We define the scalar curvature $\tau(L)$ of the $r$-plane section $L$ by

$$
\tau(L)=\sum_{1 \leq \alpha<\beta \leq r} K\left(e_{\alpha} \wedge e_{\beta}\right)
$$

For an integer $k \geq 0$, we denote by $S(n, k)$ the set of $k$-tuples $\left(n_{1}, \ldots, n_{k}\right)$ of integers $\geq 2$ satisfying $n_{1}<n, n_{1}+\cdots+n_{k} \leq n$. We denote by $S(n)$ the set of unordered $k$-tuples with $k \geq 0$ for a fixed $n$.

For each $k$-tuples $\left(n_{1}, \ldots, n_{k}\right) \in S(n)$, Chen introduced a Riemannian invariant $\delta\left(n_{1}, \ldots\right.$, $n_{k}$ ) defined by

$$
\delta\left(n_{1}, \ldots, n_{k}\right)(p)=\tau(p)-S\left(n_{1}, \ldots, n_{k}\right)(p),
$$

where

$$
S\left(n_{1}, \ldots, n_{k}\right)(p)=\inf \left\{\tau\left(L_{1}\right)+\cdots+\tau\left(L_{k}\right)\right\},
$$

$L_{1}, \ldots, L_{k}$ running over all $k$ mutually orthogonal subspaces of $T_{p} M$ such that $\operatorname{dim} L_{j}=n_{j}$, $j \in\{1, \ldots, k\}$. We note that the Chen invariant with $k=0$ is nothing but the scalar curvature. Also, we denote by $d\left(n_{1}, \ldots, n_{k}\right)$ and $b\left(n_{1}, \ldots, n_{k}\right)$ the real constants given by

$$
d\left(n_{1}, \ldots, n_{k}\right)=\frac{n^{2}\left(n+k-1-\sum_{j=1}^{k} n_{j}\right)}{2\left(n+k-\sum_{j=1}^{k} n_{j}\right)}
$$


and

$$
b\left(n_{1}, \ldots, n_{k}\right)=\frac{n(n-1)-\sum_{j=1}^{k} n_{j}\left(n_{j}-1\right)}{2} .
$$

For a $k$-plane section $L$ of $T_{p} M, p \in M$, and $X$ a unit vector in $L$, one can choose an orthonormal basis $\left\{e_{1}, \ldots, e_{k}\right\}$ of $L$ such that $e_{1}=X$. Then the Ricci curvature of $L$ at $X$, denoted $\operatorname{Ric}_{L}(X)$, is defined by

$$
\operatorname{Ric}_{L}(X)=\sum_{j=2}^{k} K\left(X \wedge e_{j}\right) .
$$

We note that such a curvature is called a $k$-Ricci curvature.

For an integer $k, 2 \leq k \leq n$, B.-Y. Chen introduced a Riemannian invariant $\Theta_{k}$ defined by

$$
\Theta_{k}(p)=\frac{1}{k-1} \inf \left\{\operatorname{Ric}_{L}(X) \mid L, X\right\}, \quad p \in M,
$$

where $L$ runs over all $k$-plane sections in $T_{p} M$ and $X$ runs over all unit vectors in $L$.

It is well known that all these above invariants have many interesting applications to several fields of mathematics (see [30]).

\subsection{Quaternionic Kähler manifolds}

The geometry of Riemannian structures, complex structures, almost contact structures, hypercomplex structures, quaternionic and Cauchy-Riemann structures belongs to the general theory of the $G$-structures, a domain of great interest in modern differential geometry, in global analysis and mathematical physics. Quaternionic manifolds correspond to the reduction of the structural group at $G L(n, H) \cdot S p(1)$. From the metric viewpoint, the most interesting is the case of quaternionic Kähler manifolds, which corresponds to the reduction of the holonomy at a subgroup of $S p(n) \cdot S p(1)$. They appear in Berger's list for possible holonomy groups of irreducible Riemannian manifolds [31]. We give in this subsection a quick review of basic definitions and properties concerning the differential geometry of manifolds endowed with quaternionic structures. For details, see [32].

Let $\bar{M}$ be a differentiable manifold and assume that there is a rank 3-subbundle $\sigma$ of $\operatorname{End}(T \bar{M})$ such that a local basis $\left\{J_{1}, J_{2}, J_{3}\right\}$ exists on sections of $\sigma$ satisfying for all $\alpha \in$ $\{1,2,3\}$ :

$$
J_{\alpha}^{2}=-\mathrm{Id}, \quad J_{\alpha} J_{\alpha+1}=-J_{\alpha+1} J_{\alpha}=J_{\alpha+2},
$$

where Id denotes the identity tensor field of type $(1,1)$ on $M$ and the indices are taken from $\{1,2,3\}$ modulo 3 . Then the bundle $\sigma$ is called an almost quaternionic structure on $M$ and $\left\{J_{1}, J_{2}, J_{3}\right\}$ is called a canonical local basis of $\sigma$. Moreover, $(\bar{M}, \sigma)$ is said to be an almost quaternionic manifold. It is easy to see that any almost quaternionic manifold is of dimension $4 m, m \geq 1$.

A Riemannian metric $\bar{g}$ on $\bar{M}$ is said to be adapted to the almost quaternionic structure $\sigma$ if it satisfies:

$$
\bar{g}\left(J_{\alpha} X, J_{\alpha} Y\right)=\bar{g}(X, Y), \quad \forall \alpha \in\{1,2,3\}
$$


for all vector fields $X, Y$ on $\bar{M}$ and any canonical local basis $\left\{J_{1}, J_{2}, J_{3}\right\}$ of $\sigma$. Moreover, $(\bar{M}, \sigma, \bar{g})$ is said to be an almost quaternionic Hermitian manifold.

If the bundle $\sigma$ is parallel with respect to the Levi-Civita connection $\bar{\nabla}$ of $\bar{g}$, then $(\bar{M}, \sigma, \bar{g})$ is said to be a quaternionic Kähler manifold. Equivalently, locally defined 1-forms $\omega_{1}, \omega_{2}$, $\omega_{3}$ exist such that we have, for all $\alpha \in\{1,2,3\}$,

$$
\bar{\nabla}_{X} J_{\alpha}=\omega_{\alpha+2}(X) J_{\alpha+1}-\omega_{\alpha+1}(X) J_{\alpha+2}
$$

for any vector field $X$ on $\bar{M}$, where the indices are taken from $\{1,2,3\}$ modulo 3 .

We remark that any quaternionic Kähler manifold is an Einstein manifold, provided that $\operatorname{dim} M>4$ (see [32-34]).

Let $(\bar{M}, \sigma, \bar{g})$ be a quaternionic Kähler manifold and let $X$ be a non-null vector on $\bar{M}$. Then the 4-plane spanned by $\left\{X, J_{1} X, J_{2} X, J_{3} X\right\}$, denoted by $Q(X)$, is called a quaternionic 4-plane. Any 2-plane in $Q(X)$ is called a quaternionic plane. The sectional curvature of a quaternionic plane is called a quaternionic sectional curvature. A quaternionic Kähler manifold is a quaternionic space form if its quaternionic sectional curvatures are equal to a constant, say $c$. It is well-known that a quaternionic Kähler manifold $(\bar{M}, \sigma, \bar{g})$ is a quaternionic space form, denoted $\bar{M}(c)$, if and only if its curvature tensor is given by (see [32])

$$
\begin{aligned}
\bar{R}(X, Y) Z= & \frac{c}{4}\left\{\bar{g}(Z, Y) X-\bar{g}(X, Z) Y+\sum_{\alpha=1}^{3}\left[\bar{g}\left(Z, J_{\alpha} Y\right) J_{\alpha} X\right.\right. \\
& \left.\left.-\bar{g}\left(Z, J_{\alpha} X\right) J_{\alpha} Y+2 \bar{g}\left(X, J_{\alpha} Y\right) J_{\alpha} Z\right]\right\}
\end{aligned}
$$

for all vector fields $X, Y, Z$ on $\bar{M}$ and any local basis $\left\{J_{1}, J_{2}, J_{3}\right\}$ of $\sigma$. We note that some interesting characterizations of quaternionic space forms were obtained in [35].

For a submanifold $M$ of a quaternion Kähler manifold $(\bar{M}, \sigma, \bar{g})$, we denote by $g$ the metric tensor induced on $M$. If $\nabla$ is the covariant differentiation induced on $M$, the Gauss and Weingarten formulas are given by

$$
\bar{\nabla}_{X} Y=\nabla_{X} Y+h(X, Y), \quad \forall X, Y \in \Gamma(T M)
$$

and

$$
\bar{\nabla}_{X} N=-A_{N} X+\nabla_{X}^{\perp} N, \quad \forall X \in \Gamma(T M), \forall N \in \Gamma\left(T M^{\perp}\right),
$$

where $h$ is the second fundamental form of $M, \nabla^{\perp}$ is the connection on the normal bundle and $A_{N}$ is the shape operator of $M$ with respect to $N$. The shape operator $A_{N}$ is related to $h$ by

$$
g\left(A_{N} X, Y\right)=\bar{g}(h(X, Y), N)
$$

for all $X, Y \in \Gamma(T M)$ and $N \in \Gamma\left(T M^{\perp}\right)$. 
If $\left\{e_{1}, \ldots, e_{n}\right\}$ is an orthonormal basis of $T_{p} M$ and $\left\{e_{n+1}, \ldots, e_{4 m}\right\}$ is an orthonormal basis of $T_{p}^{\perp} M$, where $p \in M$, we denote by $H$ the mean curvature vector, that is,

$$
H(p)=\frac{1}{n} \sum_{i=1}^{n} h\left(e_{i}, e_{i}\right)
$$

Also, we set

$$
h_{i j}^{r}=g\left(h\left(e_{i}, e_{j}\right), e_{r}\right), \quad i, j \in\{1, \ldots, n\}, r \in\{n+1, \ldots, 4 m\}
$$

and

$$
\|h\|^{2}(p)=\sum_{i, j=1}^{n} g\left(h\left(e_{i}, e_{j}\right), h\left(e_{i}, e_{j}\right)\right) .
$$

The submanifold $M$ is called totally geodesic if the second fundamental form vanishes identically and totally umbilical if there is a real number $\lambda$ such that $h(X, Y)=\lambda g(X, Y) H$ for any tangent vectors $X, Y$ on $M$. If $H=0$, then the submanifold $M$ is said to be minimal.

\section{Fundamental inequalities involving Chen invariants and the squared mean curvature}

For a Riemannian submanifold $M^{n}$ of a real space form $\bar{M}$ with constant sectional curvature $c$, Chen [1] proved the following inequality for the Riemannian invariant $\delta_{M}$ of $M$, known as the first Chen inequality:

$$
\delta_{M} \leq \frac{n-2}{2}\left[\frac{n^{2}}{n-1}\|H\|^{2}+c(n+1)\right]
$$

The submanifold $M$ is said to satisfy Chen's equality if the equality case of (15) holds identically.

In quaternionic Kähler ambient, the first classes of submanifolds which have been introduced and studied were quaternionic [36] and totally real submanifolds [37]. A submanifold $M$ in a quaternionic Kähler manifold $(\bar{M}, \sigma, \bar{g})$ is called a quaternionic submanifold (resp. a totally real submanifold) if each tangent space of $M$ is carried into itself (resp. into the normal space) by each section in $\sigma$. An $n$-dimensional totally real submanifold of a quaternionic space form $\bar{M}^{4 m}(c)$ is said to be a Lagrangian submanifold if $n=m$. It is well known that a quaternionic submanifold is totally geodesic, so the first class of interest from Chen's inequalities viewpoint is given by the totally real submanifolds. In [38], the validity of the inequality (15) was proved for totally real submanifolds in a quaternionic space form $\bar{M}(4 c)$ of quaternionic sectional curvature $4 c$, and Chen's equality was interpreted in terms of eigenvalues and eigenspaces of the Weingarten operators of the submanifold. As a consequence, Hong and Houh obtained the following interesting result.

Theorem 3.1 [38] Every Lagrangian submanifold of a quaternionic space form satisfying Chen's equality is minimal. 
In [39], Şahin introduced the concept of slant submanifolds as a natural generalization of both quaternionic and totally real submanifolds. A submanifold $M$ of a quaternionic Kähler manifold $\bar{M}$ is said to be a slant submanifold if for each non-zero vector $X \tan$ gent to $M$ at $p$, the angle $\theta(X)$ between $J_{\alpha}(X)$ and $T_{p} M, \alpha \in\{1,2,3\}$ is constant, i.e., it does not depend on choice of $p \in M$ and $X \in T_{p} M$. We can easily see that quaternionic submanifolds are slant submanifolds with $\theta=0$ and totally-real submanifolds are slant submanifolds with $\theta=\frac{\pi}{2}$. A slant submanifold of a quaternionic Käler manifold is said to be proper (or $\theta$-slant proper) if it is neither quaternionic nor totally real.

In [40], the present author obtained the generalization of the first Chen inequality to the case of slant submanifolds in quaternionic space forms as follows.

Theorem 3.2 [40] Let $M^{n}$ be a $\theta$-slant proper submanifold of a quaternionic space form $\bar{M}^{4 m}(c)$. Then, for each point $p \in M$, we have

$$
\delta_{M}(p) \leq \frac{n-2}{2}\left[\frac{n^{2}}{n-1}\|H\|^{2}+\frac{c}{4}\left(n+1+9 \cos ^{2} \theta\right)\right] .
$$

The equality in (16) holds at $p \in M$ if and only if there exists an orthonormal basis $\left\{e_{1}, \ldots, e_{n}\right\}$ of $T_{p} M$ and an orthonormal basis $\left\{e_{n+1}, \ldots, e_{4 m}\right\}$ of $T_{p}^{\perp} M$ such that the shape operators $A_{r} \equiv A_{e_{r}}, r \in\{n+1, \ldots, 4 m\}$, take the following forms:

$$
A_{n+1}=\left(\begin{array}{ccccc}
a & 0 & 0 & \cdots & 0 \\
0 & a & 0 & \cdots & 0 \\
0 & 0 & a+b & \cdots & 0 \\
\vdots & \vdots & \vdots & \ddots & \vdots \\
0 & 0 & 0 & \cdots & a+b
\end{array}\right)
$$

and

$$
A_{r}=\left(\begin{array}{ccccc}
a_{r} & b_{r} & 0 & \cdots & 0 \\
a_{r} & -b_{r} & 0 & \cdots & 0 \\
0 & 0 & 0 & \cdots & 0 \\
\vdots & \vdots & \vdots & \ddots & \vdots \\
0 & 0 & 0 & \cdots & 0
\end{array}\right), \quad r \in\{n+2, \ldots, 4 m\}
$$

The second Chen fundamental inequality, stated in [41], asserts that for any submanifold $M^{n}$ of a real space form $\bar{M}$ with constant sectional curvature $c$, we have

$$
\delta\left(n_{1}, \ldots, n_{k}\right) \leq d\left(n_{1}, \ldots, n_{k}\right)\|H\|^{2}+b\left(n_{1}, \ldots, n_{k}\right) c
$$

for any $k$-tuples $\left(n_{1}, \ldots, n_{k}\right) \in S(n)$. Immersion of a submanifold which realizes equality in this inequality at every point is said to be an ideal immersion.

In [42], Yoon proved that the inequality (19) is also true for totally real submanifolds in a quaternionic space forms of quaternionic sectional curvature $4 c$. This inequality was later generalized for slant submanifolds in quaternionic space forms as follows. 
Theorem 3.3 [40] If $M^{n}$ is a $\theta$-slant proper submanifold of a quaternionic space form $\bar{M}^{4 m}(c)$, then we have

$$
\delta\left(n_{1}, \ldots, n_{k}\right) \leq d\left(n_{1}, \ldots, n_{k}\right)\|H\|^{2}+b\left(n_{1}, \ldots, n_{k}\right) \frac{c}{4}+\frac{9 c}{8}\left(n-\sum_{j=1}^{k} n_{j}\right) \cos ^{2} \theta
$$

for any $k$-tuples $\left(n_{1}, \ldots, n_{k}\right) \in S(n)$.

The equality in (20) holds at $p \in M$ if and only if there exists an orthonormal basis $\left\{e_{1}, \ldots, e_{n}\right\}$ of $T_{p} M$ and an orthonormal basis $\left\{e_{n+1}, \ldots, e_{4 m}\right\}$ of $T_{p}^{\perp} M$ such that the shape operators $A_{r} \equiv A_{e_{r}}, r \in\{n+1, \ldots, 4 m\}$, take the following forms:

$$
A_{n+1}=\left(\begin{array}{ccccc}
a_{1} & 0 & 0 & \ldots & 0 \\
0 & a_{2} & 0 & \cdots & 0 \\
0 & 0 & a_{3} & \cdots & 0 \\
\vdots & \vdots & \vdots & \ddots & \vdots \\
0 & 0 & 0 & \cdots & a_{n}
\end{array}\right)
$$

and

$$
A_{r}=\left(\begin{array}{cccccc}
B_{1}^{r} & \cdots & 0 & 0 & \cdots & 0 \\
\vdots & \ddots & \vdots & \vdots & \ddots & \vdots \\
0 & \cdots & B_{k}^{r} & 0 & \cdots & 0 \\
0 & \cdots & 0 & c_{r} & \cdots & 0 \\
\vdots & \ddots & \vdots & \vdots & \ddots & \vdots \\
0 & \cdots & 0 & 0 & \cdots & c_{r}
\end{array}\right), \quad r \in\{n+2, \ldots, 4 m\}
$$

where $a_{1}, \ldots, a_{n}$ satisfy

$$
a_{1}+\cdots+a_{n_{1}}=\cdots=a_{n_{1}+\cdots+n_{k-1}+1}+\cdots+a_{n_{1}+\cdots+n_{k}}=a_{n_{1}+\cdots+n_{k}+1}=\cdots=a_{n}
$$

and each $B_{j}^{r}$ is a symmetric $n_{j} \times n_{j}$ submatrix satisfying:

$$
\operatorname{trace}\left(B_{1}^{r}\right)=\cdots=\operatorname{trace}\left(B_{k}^{r}\right)=c_{r} .
$$

\section{Fundamental inequalities involving the Ricci curvature and the squared mean curvature}

In [4], Chen established a sharp relationship involving the Ricci curvature and the squared mean curvature for an arbitrary $n$-dimensional Riemannian submanifold of a real space form of constant sectional curvature $c$,

$$
\operatorname{Ric}(X) \leq(n-1) c+\frac{n^{2}}{4}\|H\|^{2}
$$

which is known as the Chen-Ricci inequality. In [43], Liu obtained the same inequality for totally real submanifolds in quaternionic space forms.

A submanifold $M$ of a quaternion Kähler manifold $(\bar{M}, \sigma, \bar{g})$ is said to be a quaternionic CR-submanifold if there exists two orthogonal complementary distributions $D$ and $D^{\perp}$ on 
$M$ such that $D$ is invariant under quaternionic structure and $D^{\perp}$ is totally real (see [44]). An estimation of the Ricci curvature of a quaternionic CR-submanifold in a quaternionic space form has been established in [45], as follows.

Theorem 4.1 [45] Let $M$ be an n-dimensional quaternionic CR-submanifold of a quaternionic space form $\bar{M}(c)$. Then:

$\left(\mathrm{i}_{1}\right)$ For each unit vector $X \in D_{p}^{\perp}$, we have

$$
\operatorname{Ric}(X) \leq \frac{(n-1) c}{4}+\frac{n^{2}}{4}\|H\|^{2}
$$

(i2) For each unit vector $X \in D_{p}$, we have

$$
\operatorname{Ric}(X) \leq \frac{(n+8) c}{4}+\frac{n^{2}}{4}\|H\|^{2}
$$

(ii) If $H(p)=0$, then a unit tangent vector $X$ at $p$ satisfies the equality case of (24) (respectively (25)) if and only if $X \in D_{p}^{\perp} \cap \mathcal{N}_{p}$ (respectively $X \in D_{p} \cap \mathcal{N}_{p}$ ), where $N_{p}$ is the relative null space of $M$ at the point $p \in M$ defined by

$$
\mathcal{N}_{p}=\left\{Z \in T_{p} M \mid h(Z, Y)=0, \forall Y \in T_{p} M\right\} .
$$

We note that an optimal inequality concerning the Ricci curvature for quaternionic CR-submanifolds of quaternionic space forms with a semi-symmetric metric connection was obtained in [46]. It is clear that, although quaternionic CR-submanifolds are also the generalization of both quaternionic and totally real submanifolds, there exists no inclusion between the two classes of quaternionic CR-submanifolds and slant submanifolds. The following estimation of the Ricci curvature for slant submanifolds in quaternionic space forms was firstly proved in [40], and an alternative nice proof can be found in [47].

Theorem 4.2 [40] Let $M^{n}$ be a $\theta$-slant proper submanifold of a quaternionic space form $\bar{M}^{4 m}(c)$. Then:

(i) For each unit vector $X \in T_{p} M$, we have

$$
\operatorname{Ric}(X) \leq \frac{(n-1) c}{4}+\frac{n^{2}}{4}\|H\|^{2}+\frac{3 c}{8} \cos ^{2} \theta
$$

(ii) If $H(p)=0$, then a unit tangent vector $X$ at $p$ satisfies the equality case of (26) if and only if $X$ belongs to the relative null space of $M$ at $p$.

(iii) The equality case of (26) holds identically for all unit tangent vectors at $p$ if and only either $p$ is a totally geodesic point or $n=2$ and $p$ is a totally umbilical point.

In [48], Oprea proved using optimization methods that the inequality (23) is not optimal for Lagrangian submanifolds in complex space forms and obtained an improved Chen-Ricci inequality. In [49], Deng obtained the proof of the improved inequality as an application of suitable algebraic inequalities. This allows one to determine the equality condition in an explicit form. Recently, in [50], the Chen-Ricci inequality was improved for Lagrangian submanifolds in quaternionic space forms as follows. 
Theorem 4.3 [50] Let $M^{n}$ be a Lagrangian submanifold of real dimension $n \geq 2$ in a quaternionic space form $\bar{M}^{4 n}(c), p$ be a point in $M$ and $X$ be a unit tangent vector in $T_{p} M$. Then we have

$$
\operatorname{Ric}(X) \leq \frac{(n-1)}{4}\left(c+\|H\|^{2}\right)
$$

Moreover, the equality sign holds for any unit tangent vector at $p$ if and only if either

(i) $p$ is a totally geodesic point or

(ii) $n=2$ and $p$ is an $H$-umbilical point with $\lambda_{r}=3 \mu_{r}, r=1,2,3$, i.e., there exists an orthonormal basis $\left\{e_{1}, \ldots, e_{n}\right\}$ of $T_{p} M$ such that the second fundamental form takes the following simple form:

$$
\begin{aligned}
& h\left(e_{1}, e_{1}\right)=\sum_{\alpha=1}^{3} \lambda_{\alpha} J_{\alpha}\left(e_{1}\right), \\
& h\left(e_{2}, e_{2}\right)=\sum_{\alpha=1}^{3} \mu_{\alpha} J_{\alpha}\left(e_{1}\right), \\
& h\left(e_{1}, e_{j}\right)=\sum_{\alpha=1}^{3} \mu_{\alpha} J_{\alpha}\left(e_{j}\right), \\
& h\left(e_{j}, e_{k}\right)=0, \quad j \neq k, j, k=2, \ldots, n
\end{aligned}
$$

for some suitable functions $\lambda_{r}$ and $\mu_{r}$ satisfying $\lambda_{r}=3 \mu_{r}, r=1,2,3$.

\section{Fundamental inequalities involving $k$-Ricci curvature, squared mean curvature and shape operator}

In [4], Chen extended the notion of Ricci curvature to $k$-Ricci curvature for a Riemannian manifold and established a sharp relationship between $k$-Ricci curvatures and the shape operator and also a sharp relationship between $k$-Ricci curvatures and the squared mean curvature for an $n$-dimensional Riemannian submanifold in a real space form $\bar{M}$ with constant sectional curvature $c$. More precisely, he proved that for any point $p \in M$ and any integer $k, 2 \leq k \leq n$, one has

$$
\|H\|^{2}(p) \geq \frac{4(n-1)}{n^{2}}\left[\frac{\Theta_{k}(p)}{k-1}-c\right] .
$$

On the other hand, Chen proved that if $\Theta_{k}(p) \neq c$, then the shape operator at the mean curvature vector satisfies for any integer $k, 2 \leq k \leq n$, and any point $p \in M$,

$$
A_{H}>\frac{n-1}{n}\left[\Theta_{k}(p)-c\right] I_{n}
$$

at $p$, where $I_{n}$ denotes the identity map of $T_{p} M^{n}$.

The inequality (28) was extended in the setting of a totally real submanifold in a quaternionic space form by Liu and Dai in [51] and recently, in [52], both inequalities (28) and (29) were generalized for proper slant submanifolds in quaternionic space forms as follows. 
Theorem 5.1 [52] Let $M^{n}$ be a $\theta$-slant proper submanifold of a quaternionic space form $\bar{M}^{4 m}(c)$. Then, for any $p \in M$ and any integer $k, 2 \leq k \leq n$, one has

$$
\|H\|^{2}(p) \geq \Theta_{k}(p)-\frac{c}{4}\left(1+\frac{9}{n-1} \cos ^{2} \theta\right) .
$$

Theorem 5.2 [52] Let $x: M \rightarrow \bar{M}^{4 m}(c)$ be an isometric immersion of an n-dimensional $\theta$-slant proper submanifold $M$ into a 4 -dimensional quaternionic space form $\bar{M}(c)$. Then, for any $p \in M$ and any integer $k, 2 \leq k \leq n$, one has:

(i) If $\Theta_{k}(p) \neq \frac{c}{4}\left(1+\frac{9}{n-1} \cos ^{2} \theta\right)$, then the shape operator at the mean curvature satisfies

$$
A_{H}>\frac{n-1}{n}\left[\Theta_{k}(p)-\frac{c}{4}\left(1+\frac{9}{n-1} \cos ^{2} \theta\right)\right] I_{n},
$$

at $p$, where $I_{n}$ denotes the identity map of $T_{p} M$.

(ii) If $\Theta_{k}(p)=\frac{c}{4}\left(1+\frac{9}{n-1} \cos ^{2} \theta\right)$, then $A_{H} \geq 0$ at $p$.

(iii) A unit vector $X \in T_{p} M$ satisfies

$$
A_{H} X=\frac{n-1}{n}\left[\Theta_{k}(p)-\frac{c}{4}\left(1+\frac{9}{n-1} \cos ^{2} \theta\right)\right] X
$$

if and only if $\Theta_{k}(p)=\frac{c}{4}\left(1+\frac{9}{n-1} \cos ^{2} \theta\right)$ and $X$ belongs to $\mathcal{N}_{p}$, the relative null space of $M$ at $p$.

(iv) The identity

$$
A_{H}=\frac{n-1}{n}\left[\Theta_{k}(p)-\frac{c}{4}\left(1+\frac{9}{n-1} \cos ^{2} \theta\right)\right] I_{n}
$$

holds at $p$ if and only if $p$ is a totally geodesic point.

\section{Some open problems}

In this section we propose to investigate the following open problems related to the Chen invariants and ideal immersions in quaternionic space forms.

Problem 6.1 QR-submanifolds were introduced by Bejancu [53] as a generalization of the real hypersurfaces of a quaternionic Kähler manifold. In fact, a real submanifold $M$ of a quaternionic Kähler manifold $(\bar{M}, \sigma, \bar{g})$ is called a QR-submanifold if there exists a vector subbundle $D$ of the normal bundle $T M^{\perp}$ such that $J_{\alpha}\left(D_{p}\right)=D_{p}$ and $J_{\alpha}\left(D_{p}^{\perp}\right) \subset T_{p} M$, for all $p \in M, \alpha=1,2,3$ and for any local basis $\left\{J_{1}, J_{2}, J_{3}\right\}$ of $\sigma$, where $D^{\perp}$ is the complementary orthogonal bundle to $D$ in $T M^{\perp}$. The problem is to extend the inequalities (15), (19), (23), (28) and (29) for QR-submanifolds in quaternionic space forms.

Problem 6.2 To generalize the Theorems 3.2, 3.3, 4.1, 4.2, 5.1 and 5.2 for semi-slant submanifolds in quaternionic space forms.

We note that the concept of a semi-slant submanifold in quaternionic geometry was introduced by Şahin [39] as follows: a real submanifold $M$ of a quaternionic Kähler manifold $(\bar{M}, \sigma, \bar{g})$ is said to be a semi-slant submanifold if there exist two orthogonal vector subbundles $\mu$ and $\mu^{\perp}$ of the normal bundle $T M^{\perp}$ such that $T M^{\perp}=\mu \oplus \mu^{\perp}, \mu_{p}^{\perp}$ is antiinvariant with respect to $J_{\alpha}$ and $\mu_{p}$ is slant with respect to $J_{\alpha}$ for all $p \in M, \alpha=1,2,3$ 
and for any local basis $\left\{J_{1}, J_{2}, J_{3}\right\}$ of $\sigma$. It is easy to see that this notion is natural because QR-submanifolds and in particular real hypersurfaces are examples of semi-slant submanifolds of a quaternionic Kähler manifold and therefore fulfill the main purpose for which semi-slant submanifolds were introduced in Kähler geometry by Papaghiuc [54]. We also remark that recently, in [55], Shukla and Rao defined another concept of a semi-slant submanifold of a quaternionic Kähler manifold by analogy with the definition of Papaghiuc, but that class of submanifolds, although generalizes slant submanifolds, does not contain real hypersurfaces as a subclass.

Problem 6.3 Recently, Tripathi [56], Mihai and Rădulescu [57] obtained an improved Chen-Ricci inequality for Kählerian slant submanifolds in a complex space form. On the other hand, the quaternionic version of a Kählerian slant submanifold has been introduced in [39], under the name of a quaternionic slant submanifold, and some properties were obtained in [52]. Thus a proper slant submanifold $M$ of a quaternionic Kähler manifold $(\bar{M}, \sigma, \bar{g})$ is said to be a quaternionic slant submanifold if it satisfies the condition

$$
\left(\bar{\nabla}_{X} P_{\alpha}\right) Y=\omega_{\alpha+2}(X) P_{\alpha+1} Y-\omega_{\alpha+1}(X) P_{\alpha+2} Y
$$

for all vector fields $X, Y$ on $\bar{M}$, where the indices are taken from $\{1,2,3\}$ modulo 3 and $P_{\alpha} Y$ denotes the tangential component of $J_{\alpha} Y$. The problem is to extend the improved ChenRicci inequality (27) to quaternionic slant submanifolds in quaternionic space forms and to investigate the equality case of the inequality.

Problem 6.4 In [58], Oprea improved the inequality (15) for Lagrangian submanifolds in complex space forms and recently, in [59], Chen and Dillen obtained improved general inequalities which involve the squared mean curvature and the invariants $\delta\left(n_{1}, \ldots, n_{k}\right)$ for Lagrangian submanifolds in complex space forms, giving also necessary and sufficient condition for a Lagrangian submanifold to attain the equality for arbitrary $\delta\left(n_{1}, \ldots, n_{k}\right)$. The problem is to obtain improved general inequalities for Lagrangian submanifolds in quaternionic space forms and to completely classify Lagrangian submanifolds which realize the equality case of these inequalities.

Problem 6.5 In [52] it was proved that there do not exist quaternionic slant immersions of minimal codimension in quaternionic projective space with unfull first normal bundle. An interesting problem is to investigate the existence of quaternionic slant immersions of minimal codimension in a quaternionic projective space $P^{n}(\mathbb{H})$ satisfying the equality case of (19) such that either $n_{1}+n_{2}+\cdots+n_{k}=n$ or $n_{1}+n_{2}+\cdots+n_{k}<n$ and at least one of $n_{i}$ is 2 . We note that the answer of the corresponding problem in Kählerian geometry is negative [60].

Problem 6.6 To completely classify $\delta(2)$-ideal slant submanifolds and $\delta(2,2)$-ideal Lagrangian submanifolds in quaternionic space forms.

We note that $\delta(2)$ and $\delta(2,2)$ are the simplest non-trivial $\delta$-curvature invariants and some classification results for $\delta(2)$ and $\delta(2,2)$-ideal Lagrangian submanifolds in complex space forms were obtained in [61-64]. 
Problem 6.7 To obtain Chen-like inequalities for the Casorati curvatures of slant submanifolds in quaternionic space forms and to completely classify Casorati ideal submanifolds.

It is known that the Casorati curvature of a submanifold in a Riemannian manifold is an extrinsic invariant defined as the normalized square of the length of the second fundamental form. Moreover, this notion extends the concept of the principal direction of a hypersurface of a Riemannian manifold to submanifolds of a Riemannian manifold and it was preferred by Casorati over the traditional Gauss curvature because corresponds better with the common intuition of curvature [65]. Therefore it is of great interest to obtain optimal inequalities for the Casorati curvatures of submanifolds in different ambient spaces. We note that in [66], Decu, Haesen and Verstraelen obtained some optimal inequalities involving the scalar curvature and the Casorati curvature of a Riemannian submanifold in a real space form and the holomorphic sectional curvature and the Casorati curvature of a Kähler hypersurface in a complex space form. Moreover, the same authors proved in [67] an inequality in which the scalar curvature is estimated from above by the normalized Casorati curvatures.

Competing interests
The author declares that he has no competing interests.

Additional information

This paper is a contribution to the International Conference on Applied Analysis and Algebra (ICAAA2012) (20-24 June 2012, Istanbul, Turkey).

\section{Acknowledgements}

Dedicated to Prof. Ravi P Agarwal on his 65th birth anniversary.

This work was supported by CNCS-UEFISCDI, project number PN-II-ID-PCE-2011-3-0118.

Received: 26 September 2012 Accepted: 9 February 2013 Published: 22 February 2013

References

1. Chen, B-Y: Some pinching and classification theorems for minimal submanifolds. Arch. Math. 60, 568-578 (1993)

2. Chern, SS: Minimal Submanifolds in a Riemannian Manifold. University of Kansas Press, Lawrence (1968)

3. Suceavă, B, Vâjiac, M: Remarks on Chen's fundamental inequality with classical curvature invariants in Riemannian spaces. An. Ştiint. Univ. Al. I. Cuza laşi. Mat. 54(1), 27-37 (2008)

4. Chen, B-Y: Relations between Ricci curvature and shape operator for submanifolds with arbitrary codimensions. Glasg. Math. J. 41, 33-41 (1999)

5. Chen, B-Y: A general inequality for submanifolds in complex-space-forms and its applications. Arch. Math. 67(6), 519-528 (1996)

6. Chen, B-Y: An optimal inequality for CR-warped products in complex space forms involving CR $\delta$-invariant. Int. J. Math. 23(3), 1250045 (2012)

7. Oiagă, A, Mihai, I: B.-Y. Chen inequalities for slant submanifolds in complex space forms. Demonstr. Math. 32, 835-846 (1999)

8. Kim, J-S, Choi, J: A basic inequality for submanifolds in a cosymplectic space form. Int. J. Math. Math. Sci. 9, 539-547 (2003)

9. Liu, X, Su, W: Shape operator of slant submanifolds in cosymplectic space forms. Studia Sci. Math. Hung. 42(4), 387-400 (2005)

10. Yoon, DW: Inequality for Ricci curvature of slant submanifolds in cosymplectic space forms. Turk. J. Math. 30,43-56 (2006)

11. Cioroboiu, D, Oiagă, A: B.Y. Chen inequalities for slant submanifolds in Sasakian space forms. Rend. Circ. Mat. Palermo 52, 367-381 (2003)

12. Defever, F, Mihai, I, Verstraelen, L: B.-Y. Chen's inequality for C-totally real submanifolds of Sasakian space forms. Boll. Unione Mat. Ital, B 11(2), 365-374 (1997)

13. Mihai, I: Ricci curvature of submanifolds in Sasakian space forms. J. Aust. Math. Soc. 72(2), 247-256 (2002)

14. Carriazo, A, Kim, YH, Yoon, DW: Some inequalities on totally real submanifolds in locally conformal Kähler space forms. J. Korean Math. Soc. 41(5), 795-808 (2004)

15. Hong, S, Matsumoto, K, Tripathi, MM: Certain basic inequalities for submanifolds of locally conformal Kähler space forms. SUT J. Math. 41(1), 75-94 (2005)

16. Kim, J-S, Yoon, DW: Inequality for totally real warped products in locally conformal Kaehler space forms. Kyungpook Math. J. 44(4), 585-592 (2004) 
17. Alegre, P, Carriazo, A, Kim, YH, Yoon, DW: B.-Y. Chen's inequality for submanifolds of generalized space forms. Indian J. Pure Appl. Math. 38(3), 185-201 (2007)

18. Kim, J-S, Song, YM, Tripathi, MM: B.-Y. Chen inequalities for submanifolds in generalized complex space forms. Bull. Korean Math. Soc 40, 411-423 (2003)

19. Mihai, A: Shape operator $A_{H}$ for slant submanifolds in generalized complex space forms. Turk. J. Math. 27(4), 509-523 (2003)

20. Arslan, K, Ezentas, R, Mihai, I, Murathan, C, Özgür, C: B.Y. Chen inequalities for submanifolds in locally conformal almost cosymplectic manifolds. Bull. Inst. Math. Acad. Sin. 29(3), 231-242 (2001)

21. Yoon, DW: Inequality for Ricci curvature of certain submanifolds in locally conformal almost cosymplectic manifolds. Int. J. Math. Math. Sci. 10, 1621-1632 (2005)

22. Arslan, K, Ezentas, R, Mihai, I, Murathan, C, Özgür, C: Certain inequalities for submanifolds in $(k, \mu)$-contact space forms. Bull. Aust. Math. Soc. 64(2), 201-212 (2001)

23. Tripathi, MM, Kim, JS: C-totally real submanifolds in $(\kappa, \mu)$-contact space forms. Bull. Aust. Math. Soc. 67(1), 51-65 (2003)

24. Arslan, K, Ezentas, R, Mihai, I, Murathan, C, Özgür, C: Ricci curvature of submanifolds in Kenmotsu space forms. Int. J. Math. Math. Sci. 29(12), 719-726 (2002)

25. Carriazo, A, Fernandez, LM, Hans-Uber, MB: B.Y. Chen's inequality for S-space-forms: applications to slant immersions Indian J. Pure Appl. Math. 34(9), 1287-1298 (2003)

26. Fernández, LM, Hans-Uber, MB: New relationships involving the mean curvature of slant submanifolds in S-space-forms. J. Korean Math. Soc. 44(3), 647-659 (2007)

27. Kim, J-S, Dwivedi, MK, Tripathi, MM: Ricci curvature of integral submanifolds of an S-space form. Bull. Korean Math. Soc. 44(3), 395-406 (2007)

28. Aktan, N, Zeki Sarikaya, M, Ozusaglam, E: B.Y. Chen's inequality for semi-slant submanifolds in T-space forms. Balk J. Geom. Appl. 13(1), 1-10 (2008)

29. Chen, B-Y: $\delta$-Invariants, Inequalities of Submanifolds and Their Applications. Topics in Differential Geometry, pp. 29-155. Ed. Acad. Române, Bucharest (2008)

30. Chen, B-Y: Pseudo-Riemannian Geometry, $\delta$-Invariants and Applications. World Scientific, Hackensack (2011)

31. Berger, M: Sur les groupes d'holonomie homogène des variétés à connexion affine et des variétés riemanniennes. Bull. Soc. Math. Fr. 83, 279-330 (1955)

32. Ishihara, S: Quaternion Kählerian manifolds. J. Differ. Geom. 9, 483-500 (1974)

33. Besse, A: Einstein Manifolds. Springer, Berlin (1987)

34. Salamon, S: Differential geometry of quaternionic manifolds. Ann. Sci. Éc. Norm. Super. 19, 31-55 (1986)

35. Adachi, T, Maeda, S: Some characterizations of quaternionic space forms. Proc. Jpn. Acad., Ser. A, Math. Sci. 76(10), 168-172 (2000)

36. Gray, A: A note on manifolds whose holonomy group is a subgroup of $S p(n) \cdot S p(1)$. Mich. Math. J. 16, 125-128 (1969)

37. Chen, B-Y, Houh, CS: Totally real submanifolds of a quaternion projective space. Ann. Mat. Pura Appl. 120(1), 185-199 (1979)

38. Hong, Y, Houh, CS: Lagrangian submanifolds of quaternion Kaehlerian manifolds satisfying Chen's equality. Beiträge Algebra Geom. 39(2), 413-421 (1998)

39. Şahin, B: Slant submanifolds of quaternion Kaehler manifolds. Commun. Korean Math. Soc. 22(1), 123-135 (2007)

40. Vîlcu, GE: B.-Y. Chen inequalities for slant submanifolds in quaternionic space forms. Turk. J. Math. 34(1), 115-128 (2010)

41. Chen, B-Y: Some new obstructions to minimal and Lagrangian isometric immersions. Jpn. J. Math. 26(1), 105-127 (2000)

42. Yoon, DW: A basic inequality of submanifolds in quaternionic space forms. Balk. J. Geom. Appl. 9(2), $92-102$ (2004)

43. Liu, X: On Ricci curvature of totally real submanifolds in a quaternion projective space. Arch. Math. 38(4)، 297-305 (2002)

44. Barros, M, Chen, B-Y, Urbano, F: Quaternion CR-submanifolds of quaternion manifolds. Kodai Math. J. 4, 399-417 (1981)

45. Mihai, I, Al-Solamy, F, Shahid, MH: On Ricci curvature of a quaternion CR-submanifold in a quaternion space form Rad. Mat. 12(1), 91-98 (2003)

46. Decu, S: Optimal inequalities for submanifolds in quaternion-space-forms with semi-symmetric metric connection. Bull. Transilv. Univ. Braşov, Ser. III 2(51), 175-184 (2009)

47. Shahid, MH, Al-Solamy, F: Ricci tensor of slant submanifolds in a quaternion projective space. C. R. Math. Acad. Sci. Paris 349(9), 571-573 (2011)

48. Oprea, T: On a geometric inequality. arXiv:math.DG/0511088

49. Deng, S: An improved Chen-Ricci inequality. Int. Electron. J. Geom. 2(2), 39-45 (2009)

50. Deng, S: Improved Chen-Ricci inequality for Lagrangian submanifolds in quaternion space forms. Int. Electron. J. Geom. 5(1), 163-170 (2012)

51. Liu, X, Dai, W: Ricci curvature of submanifolds in a quaternion projective space. Commun. Korean Math. Soc. 17(4), 625-633 (2002)

52. Vîlcu, GE: Slant submanifolds of quaternionic space forms. Publ. Math. (Debr.) 81(3-4), 397-413 (2012)

53. Bejancu, A: QR-submanifolds of quaternion Kaehler manifolds. Chin. J. Math. 14, 81-94 (1986)

54. Papaghiuc, N: Semi-slant submanifolds of a Kaehlerian manifold. An. Ştiint. Univ. Al. I. Cuza laşi 40(1), 55-61 (1994)

55. Shukla, SS, Rao, PK: Ricci curvature of quaternion slant submanifolds in quaternion space forms. Acta Math. Acad. Paedagog. Nyházi. 28(1), 69-81 (2012)

56. Tripathi, MM: Improved Chen-Ricci inequality for curvature-like tensors and its application. Differ. Geom. Appl. 29(5), 685-698 (2011)

57. Mihai, A, Rădulescu, I: An improved Chen-Ricci inequality for Kaehlerian slant submanifolds in complex space forms Taiwan. J. Math. 16(2), 761-770 (2012)

58. Oprea, T: Chen's inequality in the Lagrangian case. Colloq. Math. 108, 163-169 (2007)

59. Chen, B-Y, Dillen, F: Optimal general inequalities for Lagrangian submanifolds in complex space forms. J. Math. Anal. Appl. 379(1), 229-239 (2011) 
60. Li, G, Wu, C: Slant immersions of complex space forms and Chen's inequality. Acta Math. Sci., Ser. B, Engl. Ed. 25(2), 223-232 (2005)

61. Chen, B-Y, Dillen, F, Verstraelen, L, Vrancken, L: Totally real submanifolds of $C P^{n}$ satisfying a basic equality. Arch. Math. 63(6), 553-564 (1994)

62. Chen, B-Y, Prieto-Martín, A, Wang, X: Lagrangian submanifolds in complex space forms satisfying an improved equality involving $\delta(2,2)$. Publ. Math. (Debr.) 82(1), 1-25 (2013)

63. Chen, B-Y, Vrancken, L: Lagrangian submanifolds satisfying a basic equality. Math. Proc. Camb. Philos. Soc. 120(2), 291-307 (1996)

64. Chen, B-Y, Vrancken, L: Lagrangian submanifolds of the complex hyperbolic space. Tsukuba J. Math. 26(1), 95-118 (2002)

65. Casorati, F: Mesure de la courbure des surfaces suivant l'idée commune. Ses rapports avec les mesures de courbure gaussienne et moyenne. Acta Math. 14(1), 95-110 (1890)

66. Decu, S, Haesen, S, Verstraelen, L: Optimal inequalities involving Casorati curvatures. Bull. Transilv. Univ. Braşov, Ser. B 14(49), suppl., 85-93 (2007)

67. Decu, S, Haesen, S, Verstraelen, L: Optimal inequalities characterising quasi-umbilical submanifolds. JIPAM. J. Inequal. Pure Appl. Math. 9(3), Article 79 (2008)

doi:10.1186/1029-242X-2013-66

Cite this article as: Vîlcu: On Chen invariants and inequalities in quaternionic geometry. Journal of Inequalities and Applications 2013 2013:66.

\section{Submit your manuscript to a SpringerOpen ${ }^{\ominus}$ journal and benefit from:}

- Convenient online submission

- Rigorous peer review

- Immediate publication on acceptance

- Open access: articles freely available online

- High visibility within the field

- Retaining the copyright to your article 